\title{
Gender, Sex, Sex Differences, Doping in Athletic Performance
}

This year's International Association of Athletic Federations World Athletics Championship in Doha, Qatar, provides interesting challenges. Doha is in the desert. Most athletes will compete in a fully climatized stadium guaranteeing comfortable ambient temperatures. Starting times of the remaining "real outdoor events" marathon and $20-$ or $50-\mathrm{km}$ race walk lasting between 1:30 and 4:30 h:min will be contested at 23:59 and 23:30 h:min. These events will be undertaken in challenging conditions, near $30^{\circ} \mathrm{C}$, at cost of weird timing with respect to circadian rhythm and normal life events. Commencing September 26 to October 6, 2019, this championship requires peak performances at the end of an elongated season-a challenge for qualification strategies and periodization. The latter may partly explain why recent headlines on athletics seem to focus on gender, sex, and intersex rather than exceptional athletic performances.

Sex is genetically determined expressing a specific facet of chromosomal, gonadal, hormonal, and phenotypic binary items. Most elite sports, particularly events highly dependent on physique, have distinguished categories for the sexes. This format gives women a chance to win, because on average women have lower body and muscle mass and strength, lower bone mass, and lower oxygen-transport capacity than men. These differences result in $\sim 10 \%$ to $12 \%$ better performances in running and swimming events and up to $20 \%$ higher results in jumping events in men. The winning margin defined as the difference in a performance missing a gold medal, any medal, or making the final in athletics or swimming

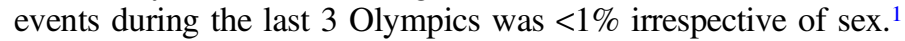

Distinction of sex appears straightforward already at birth. At birth, supposedly clear sex diagnoses may be wrong due to conflicting binary items constituting an intersex state. Named disorders (or differences) of sex development (DSD), such conditions may impose ambiguous genitalia at birth and later manifest as undervirilized males or virilized females. Depending on definitions, the frequency of DSD is debated as being between $0.018 \%$ and $1.7 \% .^{2}$ Just as a comparison, the global fraction of gingerhaired people approximates $1 \%$ to $2 \% .^{3}$

A major factor for the sex differences in performance is a well-established dose-response relationship between circulating testosterone and muscle mass, strength, and hemoglobin level. Sex differences in performance manifest from puberty onward, when circulating testosterone concentrations become 15 - to 20-fold higher than in prepubertal children, irrespective of sex, or in premenopausal women $\left(95 \%\right.$ reference ranges of $7.7-29.4 \mathrm{nmol} \cdot \mathrm{L}^{-1}$ vs $\left.0-1.7 \mathrm{nmol} \cdot \mathrm{L}^{-1}\right)$. Exogenous testosterone induces the same biological and clinical effects as endogenous testosterone, except spermatogenesis. Therefore, testosterone counts as a highly potent doping substance. Women with the mild form of hyperandrogenism caused by polycystic ovary syndrome and testosterone concentration $<5.0 \mathrm{nmol} / \mathrm{L}$ are overrepresented in elite athletics.

Gender defines individual orientation or role. It is selfidentified based on biological, psychological, and societal factors. Gender may be consistent or not with biological sex as assigned at birth. It may impose as a single, multiple, none, or even variable expression of self-identified gender. DSD can cause circulating testosterone levels comparable to those of men in persons who were raised and identify themselves as women. Some conditions of DSD are 140-fold more prevalent among elite female athletes than expected in the general population.

In athletics, there is a long history of concerns about men winning medals in female events. One historical case was Stella Walsh, the world's fastest woman in the 1930s, diagnosed postmortem with mosaicism. More recent debates including court cases concerning South African 800-m World and Olympic champion Caster Semenya or the Indian sprinter Dutee Chand, indicate there are still issues.

In 2011, the International Association of Athletic Federations established that for competing in women's events, hyperandrogenic women must be legally recognized as female and, unless they present with complete androgen insensitivity, maintain serum testosterone levels $<10 \mathrm{nmol} \cdot \mathrm{L}^{-1}$. This limit was ruled out by the Court for Arbitration in Sports in 2015 because the limit of $<10 \mathrm{nmol} \cdot \mathrm{L}^{-1}$ was based on insufficiently sound reference data. Since May 2019, the policy is that DSD-caused supranormal testosterone concentrations must be pharmaceutically reduced to $<5 \mathrm{nmol} \cdot \mathrm{L}^{-1}$ over a period of 6 months to competing in international women's running events of $400 \mathrm{~m}$ to a mile.

From an exercise physiologist's perspective, this focus on long-sprint and middle-distance events and the 6-month control period triggers some curiosity because

- Qualification standards of all disciplines reflect the wellknown performance differences between males and females. ${ }^{4}$

- Sex differences in throwing events are almost twice as high as in all running events.

- A 140-fold prevalence of some conditions of DSD among elite female athletes may not only concern one specific middledistance medal contender.

- It is unknown to what extent a 6-month suppression of testosterone concentrations to high normal female levels in elite female athletes reflects modulated muscle mass, strength, and performance as intended if chemically castrated male clinical patients show corresponding progressive losses up to 24 months.

The latter may open up a possibly even more controversially perceived issue:

What is the effective duration of a doping-related ban on competition to not only cause losses in reputation and financial income but also prevent a perpetuating benefit on muscle mass and bone structure after previously successful steroid abuse irrespective of sex?

Neither from a science perspective nor by sport administration have such areas of controversy been addressed in a sufficiently rigorous way. This challenge strongly underpins the high demand of multidisciplinary research of sport physiologists, basic scientists, clinicians, and performance specialists combining rigorous science with reasoned practical applications in elite sports.

\section{Ralph Beneke, IJSPP editor emeritus,} and Renate M. Leithäuser 


\section{References}

1. Handelsman DJ, Hirschberg AL, Bermon S. Circulating testosterone as the hormonal basis of sex differences in athletic performance. Endocr Rev. 2018;39(5):803-829. PubMed ID: 30010735 doi:10. 1210/er.2018-00020

2. Fausto-Sterling A. Sexing the Body: Gender Politics and the Construction of Sexuality. New York: Basic Books; 2000. ISBN 9780465077144.
3. Barnes H. How many redheads are there in the world? $B B C$ News. October 2, 2013. https://www.bbc.com/news/magazine24331615

4. IAAF World Athletics Championships Doha QAT, September 27 to October 6, 2019. Qualification System and Entry Standards. IAAF Council; December 2018. https://www.fla.lu/criteres/2019/iaafworld-championships-doha-2019.pdf 